\title{
El Gran Miedo de 1919 a escala global: la Semana Trágica argentina y los archivos norteamericanos $^{1}$
}

\section{The Great Fear of 1919 on a Global Scale: Argentina's Tragic Week and the North American Archives}

\author{
Daniel Lvovich ${ }^{2}$
}

\begin{abstract}
Resumen
En este artículo inscribimos los sucesos de la Semana Trágica de 1919 en una perspectiva trasnacional, al considerar los modos en que la diplomacia y los servicios de inteligencia norteamericanos la analizaron e interpretaron y vincularla con un conjunto de eventos y procesos coetáneos. De tal modo, articulamos la interpretación de la Semana Trágica como resultado de un Gran Miedo de las élites argentinas y sudamericanas con una consideración a una escala mayor acerca de los modos en que el miedo a la revolución impactaba en las conductas e interpretaciones de actores políticos relevantes de los Estados Unidos. Basamos nuestro estudio en un conjunto de informes diplomáticos y de inteligencia de los Estados Unidos que relevamos en los National Archives and Record Administration (NARA) y en el Fondo Presidente Woodrow Wilson de la Biblioteca del Congreso de los Estados Unidos.
\end{abstract}

\begin{abstract}
In this article we inscribe the events of the Tragic Week of 1919 in a transnational perspective, considering the ways in which U.S. diplomacy and intelligence services analyzed and interpreted it and linked it to a set of contemporary events and processes. Thus, we articulated the interpretation of Tragic Week as the result of a Great Fear of Argentine and South American elites with a consideration on a larger scale of the ways in which the fear of revolution impacted the behaviors and interpretations of relevant U.S. political actors. We based our study on a set of U.S. diplomatic and intelligence reports that we relied on in the National Archives and Record Administration (NARA) and the U.S. Library of Congress' Woodrow Wilson President's Fund.
\end{abstract}

\footnotetext{
${ }^{1}$ Trabajo recibido el 01/09/2019. Aceptado el 23/11/2019.

${ }^{2}$ Universidad Nacional de General Sarmiento. Consejo Nacional de Investigaciones Científicas y Técnicas. Contacto: dlvovich@ungs.edu.ar
} 
Palabras clave: Semana Trágica; Historia trasnacional; Miedo; Argentina; Estados Unidos
Keywords: Tragic Week; Transnational history; Fear; Argentina; United States

\section{Introducción}

En este artículo inscribimos los sucesos de la Semana Trágica de 1919 en una perspectiva trasnacional, al considera los modos en que la diplomacia y los servicios de inteligencia norteamericanos la analizaron e interpretaron y vincularla con un conjunto de eventos y procesos coetáneos. De tal modo, articulamos la interpretación de la Semana Trágica como resultado de un Gran Miedo de las élites argentinas y sudamericanas con una consideración a una escala mayor acerca de los modos en que el miedo a la revolución impactaba en las conductas e interpretaciones de actores políticos relevantes de los Estados Unidos. Basamos nuestro estudio en un conjunto de informes diplomáticos y de inteligencia de los Estados Unidos que relevamos en los National Archives and Record Administration (NARA) y en el Fondo Presidente Woodrow Wilson de la Biblioteca del Congreso de los Estados Unidos.

Los acontecimientos de lo que conocemos como la Semana Trágica, denominación que se refiere al ciclo de huelgas, protestas, represión y persecución desarrollado en Buenos Aires en la primera quincena de enero de 1919, han sido tradicionalmente estudiados como parte de una historia básicamente nacional. Los factores a considerar en esa perspectiva eran la naturaleza de la huelga general, la actitud del gobierno de Yrigoyen, los posicionamientos de los conservadores, el proceso de toma de decisiones sobre la represión, etc. ${ }^{3}$ Hace algunos años analizamos la Semana Trágica como una manifestación de terror colectivo de las clases dominantes argentinas (Lvovich, 2003). Aunque los distintos abordajes historiográficos previos se habían referido invariablemente a la existencia de una situación de temor a la revolución en aquellos sectores, ninguno de ellos había colocado en el centro de su análisis tal factor. Por nues-

\footnotetext{
${ }^{3}$ Entre una muy amplia producción bibliográfica, las principales obras sobre la semana trágica son: Babini (1967), Godio (1986), Rock (1972), Bilsky (1984). Sobre la Semana Trágica en el interior del país, Tur Donati (1993). Para un estado de la cuestión, cf. Falcón y Monserrat (1998). Para una selección de fuentes, Seibel (1999). Sobre los aspectos de la persecución antisemita en enero de 1919: Solominsky (1971), Mirelman (1975), Avni (1982).
} 
tra parte, habíamos sostenido que la Semana Trágica constituye un caso testigo capaz de mostrar la potencia que las representaciones sobre el enemigo pueden imprimir a las prácticas, una vez que la convicción sobre la peligrosidad de sus intenciones se apodera de los sectores dominantes de la sociedad. En este sentido, el análisis de este fenómeno resulta central a la hora de considerar la efectividad de las teorías del complot como fuente de inspiración de las prácticas sociales. En efecto, en un contexto internacional signado por las revoluciones sociales europeas, el aumento de la conflictividad social en Buenos Aires sería interpretado por los sectores dominantes como la puesta en marcha de un complot maximalista. La presencia de un segmento del movimiento obrero que manifestaba su adhesión al bolchevismo sería considerada, pese a lo limitado de su influencia, como una demostración adicional de la existencia de tal complot. La atribución - absolutamente injustificada- de una relación de causalidad entre ambos fenómenos explica el desarrollo y la difusión del terror a la revolución. A su vez, el establecimiento de tal relación de causalidad resulta incomprensible sin recordar dos modos de explicación ya tradicionales: la acusación conservadora -vigente desde hacía décadas, de hecho desde la época de la Comuna de Paris- a los «agitadores extranjeros» de ser responsables de las luchas obreras y las creencias de tipo conspirativo difundidas por la Iglesia católica. La reacción obrera ante la brutal represión policial se tomaría como un indicio más de la existencia de la conspiración, que sería confirmada por la puesta en circulación de documentos apócrifos que fueron tomados como testimonios contundentes sobre su real entidad. En este contexto se inscriben las persecuciones y la violencia ejercida contra personas y organizaciones judías, a las que se caracterizaba como responsables de los sucesos no sólo como resultado del desplazamiento semántico entre los conceptos de ruso y judío -factor tantas veces postulado como explicación- sino también a causa de unas representaciones conspirativas de larga data sobre el judaísmo que alcanzaron verosimilitud al calor de los sucesos.

No era aquella la primera ocasión en que el terror a la revolución social se apoderaba de las clases dominantes argentinas. Ya en abril de 1901 se informaba sobre la existencia de un complot anarquista internacional, versión según la cual un anarquista de Rosario habría de asesinar al Emperador Guillermo de Alemania, mientras durante la huelga marítima de 1902 las clases altas se Buenos Aires vivieron momentos de pánico cuando corrió el rumor de que treinta mil obreros armados avan- 
zaban sobre el centro de la ciudad dispuestos a llegar a la Casa de Gobierno (Oved, 1978). A diferencia de aquellas ocasiones, en las que los temores -y los rumores que actuaban como principal instrumento de su difusión- eran rápidamente desmentidos, en los primeros días de 1919 estos ya estaban sedimentados, tras un considerable período en que las versiones alarmistas se habían desplegado. En efecto, el temor a la revolución se había apoderado de las clases dominantes argentinas ya a fines de 1918, por lo que resulta preciso analizar los sucesos de enero de 1919 como una consecuencia de las creencias difundidas por entonces. A esta situación de terror colectivo alimentados por los rumores, y que se potenciaba con el modo en que era interpretada cada acción del movimiento obrero la llamamos, «el gran miedo de 1919» (Lvovich, 2003, p. 133). Esta denominación se inspira en un texto fundacional, El Gran Terror de 1789 de Georges Lefebvre, que constituye sin duda un modelo magistral para el estudio de las situaciones de pánico colectivo y sus efectos. ${ }^{4}$ Esta obra muestra el modo en que, una vez instalado en el imaginario un temor colectivo, este funcionará como principio explicativo de una multiplicidad de prácticas que, a su vez, reforzarán la creencia en la potencia del motivo del terror. La entidad fantasmática que origina el pánico se muestra entonces particularmente eficaz, a la vez como expresión de los temores y como factor que determina la dinámica de los acontecimientos que provoca.

En nuestra investigación habíamos dado cuenta de diversos elementos de orden internacional que impactaron en los modos en que el

\footnotetext{
${ }^{4}$ Lefebvre (1982). Recordemos las líneas centrales del argumento de este clásico texto. En julio de 1789 en distintas zonas rurales de Francia se propagaron versiones según las cuales un conjunto de bandidos, soldados, o ejércitos extranjeros estarían avanzando, saqueando los pueblos y exterminando a la población a su paso. Todas estas tropas estarían, según el rumor, al servicio de un complot aristocrático destinado a castigar al pueblo. La presencia de vagabundos y mendigos en los caminos, junto a la existencia del bandidismo rural, se interpretaría como una prueba tangible del complot. Ante el supuesto peligro, los campesinos en armas salieron a los caminos y las aldeas al encuentro de los enemigos, desplazamientos que reforzaron el pánico colectivo, llevando el temor a los pueblos. Mientras el asedio campesino a los castillos señoriales se multiplicaba a lo largo del país, exigiendo en ocasiones por la fuerza el cumplimiento de sus demandas, la circulación de manifiestos atribuidos al rey en los que se denunciaba el complot aristocrático reforzaban la creencia en la conjura, que se reforzaba, a la vez, con las noticias sobre los ataques a los castillos. De tal modo, pese a que el complot aristocrático jamás tuvo lugar, la creencia en su existencia provocó efectos que eran interpretados, a la vez, como prueba de su real entidad. La existencia del bandidismo rural era verídica, tanto como la de los disturbios campesinos. La atribución de sus causas al imaginario complot explica la difusión del Gran Terror.
} 
gobierno y la elite argentina interpretaron la situación de enero de 1919. Entre ellos destacábamos las noticias sobre la Revolución Rusa, la oleada insurreccional europea de 1918 y 1919 y el establecimiento de las efímeras repúblicas de Baviera, Munich y Hungría, que llegaron a un escenario argentino signado, como hemos visto, por una creciente conflictividad social. Dichas noticias aumentaron la alarma de la elite, entusiasmaron a amplios sectores de izquierda y redoblaron la difusión del discurso que vinculaba comunismo y judaísmo como parte de la perspectiva antisemita difundida por medios de prensa, sacerdotes e intelectuales católicos. Sin embargo, no eran los católicos los únicos que antes de la Semana Trágica atribuían a los judíos la participación en un complot revolucionario. En sus memorias, el por entonces embajador norteamericano en Buenos Aires Frederick Jessup Stimson (1931) afirmaba haber recibido información acerca de la existencia en 1918 y 1919 de un movimiento internacional liderado por el comunismo, que debía estallar simultáneamente en los cinco puertos más importantes para los aliados - Estocolmo, Rotterdam, Liverpool, Nueva York y Buenos Aires- y cuyos dirigentes eran en su mayoría judíos.

Otro elemento a considerar para comprender el 1919 argentino es su dimensión sudamericana. Una mirada atenta a la escala trasnacional de los procesos de protesta de masas, y de su represión, permite constatar que fenómenos similares o conexos se desarrollaron de modo simultáneo en otros países del Cono Sur. Ello posibilita el desarrollo de un análisis que, atento a esa escala, de cuenta de las similitudes en el impulso de estos fenómenos tanto como de las peculiaridades que los contextos nacionales imponen en cada caso, para comprender la conflictividad social y las diversas represiones que se desarrollaron entre fines de 1918 y enero de 1919 en Argentina, Brasil, Chile y Uruguay (Lvovich, 2016).

En todos estos casos, el temor tiene un rol explicativo central para dar cuenta de estos procesos. En esos países y por motivos socioeconómicos endógenos, desde 1917 se intensificó el conflicto social y estallaron oleadas de huelgas y movilizaciones. En todos los casos también, segmentos del movimiento obrero mostraron su entusiasmo por la Revolución Rusa, mientras sectores de las clases dominantes -al igual que buena parte de las elites de Occidente, atemorizadas por los sucesos de octubre de 1917- interpretaron esas movilizaciones sociales como el simple resultado de los activistas en las sombras, a los que asignaron diversos sentidos y pertenencias, de acuerdo a la situación y tradiciones intelectuales de cada una de las naciones sudamericanas. Un resultado de la 
amplia conflictividad social desatada fue la puesta en marcha de relevantes reformas sociales.

El temor de las élites no provenía solo de las revoluciones europeas contemporáneas, sino también de la observación de los procesos de movilización social que se desplegaban en los países vecinos, así como de las interpretaciones que de dichos sucesos daban los sectores dominantes y la mayor parte de la prensa. Un sector de las elites atribuía el conflicto social a la circulación internacional de activistas, pero no advertía que sus marcos interpretativos y los mitos conspirativos que en ocasiones los inspiraban, también eran resultado de la circulación internacional. En tal sentido, es posible afirmar que la Semana Trágica argentina resulta una expresión particular que se inserta en una serie de fenómenos relativamente similares que afectaron a los otros países del Cono Sur, a la vez insertos en un contexto mundial socialmente convulsionado. Como toda manifestación de esta naturaleza, existen elementos internacionales que ejercen influencias similares, pero se reflejan en condiciones nacionales que determinan sus particularidades. Si entre los primeros se encuentran similares configuraciones de las elites, con un ala reformista y otra inflexiblemente represiva, o marcados paralelismos en los ciclos económicos de los países considerados, podemos enumerar entre las peculiaridades la enorme violencia represiva desplegada en Argentina y Chile, que contrasta con las relativamente moderadas formas que adquirió en Brasil, o los fuertes matices antisemitas de la interpretación -y la acción gubernamental- en los países rioplatenses. Igualmente, las tradiciones nacionales explican el lugar que el supuesto responsable del mal asume en cada caso: rusos, judíos, peruanos, argentinos eran los artífices del complot acorde a los temores y representaciones de quienes los acusaban. Debemos señalar asimismo una constatación relevante en relación con la circulación de los temores y los mitos conspirativos que explicaron --al menos por un tiempo y para una parte de los sectores dominantes y de la gran prensa- los fenómenos sociales del trimestre considerado. Aunque no deja de ser cierto que esos temores provenían en buena medida de la imagen que se hacían de Moscú y Petrogrado, hemos constatado que también se originaban en el modo en que se representaban los acontecimientos de Rio de Janeiro, Santiago de Chile o Buenos Aires, o en la atribuida presencia de activistas provenientes no ya solamente de Rusia sino de los países vecinos. 


\section{Los archivos norteamericanos: El gran miedo en contexto}

La información provista por los archivos norteamericanos nos permite considerar a la Semana Trágica argentina en el marco de una serie de temores de alcance global, así como contar con nuevas evidencias para comprender las peculiaridades de los sucesos que se desarrollaron en Buenos Aires y otras ciudades.

Las comunicaciones que el embajador Stimson enviaba al departamento de Estado dan cuenta de una perspectiva en la que el temor a la revolución resultaba una constante a partir del estallido de la revolución rusa. La inspiración de esos movimientos podía provenir en la perspectiva de Stimson, y como veremos, en la del propio Departamento de Estado, bien de Moscú o bien de Berlín, dado el enfrentamiento entre Estados Unidos y Alemania en la Primera Guerra Mundial. No se puede dejar de considerar al respecto que desde la Revolución Rusa y en particular a partir del año 1919 la sociedad norteamericana atravesaba un autentico «temor rojo» (Murray, 1955), un red scare que ha sido definido como «un período breve pero intenso de intolerancia política y represión de comunistas, radicales y otros no conformistas, no fue un incidente aislado sino parte de una tradición estadounidense más amplia» (Schmidt, 2000, p. 24). Con un origen en el que se combinan loa elementos espontáneos con el aprovechamiento de las autoridades, el red scare fue «...en el fondo, un ataque a los movimientos por cambios y reformas sociales y políticas, en particular a los trabajadores organizados, los negros y los radicales, por las fuerzas del statu quo» (Schmidt, 2000, p. 26). De modo que la percepción de los diplomáticos y miembros de los servicios de inteligencia norteamericanos no expresaban solo visiones personales sino que reflejaban tendencias sociales y políticas más amplias de la sociedad estadounidense.

Ya en noviembre de 1917, Stimson daba cuenta en su informe a Washington del rumor de que a fin de diciembre o comienzos de enero del año siguiente, para la época del transporte de los cultivos, habría una huelga ferroviaria de un alcance nunca visto antes. Se sospechaba que tras esta amenaza de huelga y tras otros movimientos huelguísticos estaba el gobierno alemán. ${ }^{5}$ La preocupación de Stimson por las huelgas

\footnotetext{
${ }^{5}$ NARA II, Dept of State records re to internal affairs of Argentina, 1910-1929, Microfilmn 514, Roll22.
} 
de los estibadores y trabajadores marítimos era un resultado de su defensa de los intereses de las navieras norteamericanas. No era poco habitual que junto a la diplomacia británica, presionaran al gobierno de Yrigoyen en demanda de represión de los movimientos huelguísticos.

Considerando una perspectiva más amplia de las políticas estadounidenses, si se analizan los archivos del departamento de Estado norteamericano y los del presidente Wilson, se comprenderá que a fines de 1918 y comienzos de 1919 la preocupación norteamericana se centraba en dos temas. Por un lado, la Revolución Rusa, la guerra civil y la intervención occidental que la sucedieron y la posibilidad de que acontecimientos similares ocurrieran en otras latitudes. El seguimiento de las huelgas en todo el mundo, del movimiento de dinero y personas proveniente de Rusia y por supuesto de la revolución espartaquista era permanente en los cables diplomáticos y de inteligencia. Así, la inteligencia norteamericana examinaba con preocupación las huelgas de enero de 1919 en La Habana, París, Bruselas, Barcelona, Bilbao, Oslo, Zúrich o Glasgow y señalaba el 20 de enero -tras anunciar en diversas ocasiones la inminencia de un estallido en Holanda- que «la revolución que había sido planeada para hoy no ocurrió, La Haya, Roterdam y Amsterdam están tranquilos». ${ }^{6}$ En febrero de ese año informaban a las autoridades norteamericanas que 14 comisarios bolcheviques rusos viajaban con pasaportes daneses falsos e intentan llegar a Brasil.

Por otro lado, la información se concentraba en las conversaciones de paz en Francia y en lo que consideraban maniobras alemanas para tratar de obtener una situación más ventajosa, atacar los intereses aliados y afectar la conferencia de paz. A menudo ambos factores se coaligaban en la mirada norteamericana. Así, por ejemplo, en una comunicación de enero de 1919 se afirmaba que tanto los bolcheviques como el gobierno alemán explicaban el estallido de conflictos sociales en Francia, mientras en los cables diplomáticos relativos a las huelgas de Cuba de enero de 1919 se sostenía que el partido liberal y el gobierno alemán atizaban el conflicto. ${ }^{7}$ El Secretario de Estado Lansing, creyendo o no en la colusión entre bolcheviques y alemanes explotaba esta acusación, promoviendo para ello publicación de libelos como el Folleto: The German

\footnotetext{
${ }^{6}$ Biblioteca del Congreso de los Estados Unidos. Fondo Presidente Woodrow Wilson. Rollo de microfilm. Telegramas de inteligencia. Rollo 471.

${ }^{7}$ Biblioteca del Congreso de los Estados Unidos. Fondo Presidente Woodrow Wilson. Rollo de microfilm 471, 17 y 19 de enero de 1919
} 
Bolsheviki Conspiration. ${ }^{8}$ Entre otros usos, este folleto fue enviado a funcionarios rusos del régimen depuesto con fines de propaganda. ${ }^{9}$

No sorprende entonces que en el parte diario de inteligencia que se enviaba al presidente Wilson apareciera, en la sección dedicada al bolchevismo, una información que daba cuenta del rumor según el cual se contratarían a oficiales alemanes como instructores para el ejército argentino, lo que nos brinda un indicio acerca de la centralidad de dicha asociación hasta en los más altos niveles del gobierno estadounidense. ${ }^{10}$

Pasada la Semana Trágica, Stimson insistía en este argumento en un telegrama del 15 de febrero al secretario de Estado. Decía el embajador norteamericano que la huelga de portuarios que se desarrollaba no era de origen local sino que fue instigada y financiada por algunas agencias de Alemania y Rusia, que usaban la agitación laboral para destruir los intereses aliados a lo largo del mundo y afectar el desarrollo de las conversaciones de paz. El diplomático estaba seguro de que la que la huelga no fue declarada hasta no recibir la orden desde Alemania, pese a que las empresas navieras concedieron a los sindicatos todo lo que demandaban.

Las preocupaciones por la injerencia de Moscú y Berlín motivaron la creación de una red de espionaje sostenida por las embajadas de Francia, Italia, Inglaterra y Estados Unidos para investigar al movimiento obrero de Argentina y Uruguay y combatir al maximalismo, que desarrolló sus actividades entre fines de 1918 y mayo de 1919. El objetivo central de la red FABI - por las siglas de los países integrantes- era controlar a los maximalistas «conglomerado impreciso que abarca a los anarquistas que utilizan métodos de acción directa, a los socialistas de izquierda y a los rusos que se consideran solidarios con el gobierno revolucionario de su país» (Díaz, 2019, p. 31). Las centrales obreras quedaron fuera de esta vigilancia aunque algunas organizaciones sindicales de actividades directamente vinculadas a los intereses angloamericanas fueron objeto de control. Los responsables de la red partían del supuesto de que el movimiento revolucionario no tenía una voluntad independiente, sino que recibían directivas y financiación de Alemania.

\footnotetext{
${ }^{8}$ War information series. The Commite on Public Information, octubre de 1918.

9 Biblioteca del Congreso de los Estados Unidos. Fondo Presidente Woodrow Wilson. Rollo de microfilm 471. Telegrama de la inteligencia militar, Christiania, 4 de diciembre de 1918.

${ }^{10}$ Biblioteca del Congreso de los Estados Unidos. Fondo Presidente Woodrow Wilson. Rollo de microfilm 469. Sumario diario de inteligencia, 4 de abril de 1919.
} 


\section{La información norteamericana sobre la Semana Trágica argentina}

En las fuentes norteamericanas se constata que los telegramas originados en Buenos Aires con información de inteligencia eran firmados por el agregado militar Alfred Smith, uno de los miembros de la red. Desde fines de 1918 Smith enviaba informaciones acerca del aumento de la conflictividad social en Argentina, señalando que en los conflictos de Rosario y Mendoza se registraba la presencia de agitadores anarquistas y maximalistas. ${ }^{11}$ Sería el propio Alfred Smith quien enviaría los partes de inteligencia con los que se informó al gobierno norteamericano sobre el devenir de la Semana Trágica.

Existieron importantes divergencias interpretativas entre Smith y el embajador Frederic Stimson, quien «no era del todo idóneo para las tareas de inteligencia», por lo que sus comunicaciones tenían tonos distintos y a veces contradictorios (Díaz, 2019, p. 24). Ello resulta central para evaluar el modo en que el temor a la revolución impactaba en las lecturas e interpretaciones de los distintos actores.

Gracias a una comunicación de Stimson sabemos que un actor central de la política argentina participaba desde temprano del temor a la revolución. El 30 de noviembre de 1918 el presidente Hipólito Yrigoyen manifestó al agregado comercial de la embajada de Estados Unidos que estaba seriamente preocupado por la posibilidad de un levantamiento maximalista en Argentina. ${ }^{12}$ Esta información resulta relevante en tanto nos muestra a un presidente preocupado por un estallido revolucionario desde el período previo y que, por lo tanto, no fue sorprendido por los acontecimientos.

En sus comunicaciones al Departamento de Estado de enero y de febrero de 1919, el embajador estadounidense en Argentina Frederic Jesup Stimson mostraba su convicción de que los acontecimientos de enero se habían visto instigados tanto por Alemania -interesada en afectar a los aliados en el momento de la firma del armisticio y el desarrollo de los tratados de Paz- como por la Rusia soviética. El embajador señalaba la responsabilidad de Hipólito Yrigoyen en los acontecimientos, dada

\footnotetext{
${ }^{11}$ Biblioteca del Congreso de los Estados Unidos. Fondo Presidente Woodrow Wilson. Rollo de microfilm 471. Partes de inteligencia. Telegramas de los días 17 y 30 de diciembre de 1918.

12 NARA II, Dept of statereccs re to internalaffairs of Argentina, 1910- 1929, Microcopie 514, Rollo 22.
} 
lo que consideraba una débil actitud frente al movimiento obrero, pero estaba convencido de encontrarse frente a un complot maximalista.

En un mensaje del 11 de enero, cuando la policía de Buenos Aires informó que se había «descubierto el soviet de Buenos Aires» compuesto por judíos rusos y presidido por el militante socialista Pedro Wald noticia que no por falsa dejo de resultar creíble por un tiempo-Stimson no se sumó a la idea de que la temida revolución estaba inspirada por Moscú, sino que apelaba en su interpretación al temor a la influencia germana oculta «Los nombres muestran que escondidos tras los elementos de la anarquía hay una preponderancia en esta situación de la influencia pro-alemana». Pocos días después informaba a Washington que si la voluntad maximalista de asumir un gobierno revolucionario era incuestionable, la denuncia de un soviet encabezado por Pedro Wald parecía haber sido una versión echada a rodar por los líderes del gobernante partido radical.

Stimson confirmaba su información acerca de la existencia de una inspiración maximalista del movimiento en diálogo con Eugene Stein, antiguo embajador zarista en Buenos Aires que permanecía en Argentina. Steinle confirmó al embajador norteamericano el origen bolchevique del levantamiento e informó que el gobierno bolchevique enviaba dinero para fomentar la revolución en Estados Unidos. No se trataba, sin duda, de una fuente imparcial y confiable.

Stimson señalaba como una prueba de la existencia de un complot que de los ciento ochenta y dos muertos en lo que definía como los «primeros combates del levantamiento», ciento cincuenta de los cadáveres recogidos por las autoridades en las calles correspondían a judíos rusos. ${ }^{13}$ Ese mismo día, en un telegrama a su gobierno, el embajador transmitía las estimaciones oficiales que calculaban las bajas entre los manifestantes en 500 muertos y 550 heridos, pero dos semanas después, en una comunicación del 27 de enero, informaba al Departamento de Estado que el número de muertos en el «levantamiento» era de 1356 y el de heridos de alrededor de 5.000. ${ }^{14}$

\footnotetext{
${ }^{13}$ The part of maximalism in the disturbances of january 9, 10 and 11th, in Buenos Aires and other Plaves of the Argentine Republic, Enclosure 1 in Despatch $N^{\circ} 7421$, del Embajador Frederic Stimson al Departamento de Estado, 13 de enero de 1919, en: NARA II, Records of the Dept. of state re to internal affairs of Argentina, 1910-1929. 835.00/66216, microcopy $\mathrm{N}^{\circ} 514$, Roll 3.

14 Telegramas de los días 13 y 27 de enero de 1919, en NARA II, Records of the Dept. of state re to internal affairs of Argentina, 1910-1929. 835.00/66-216, microcopy $\mathrm{N}^{\circ} 514$, Roll 3.
} 
En contraste, los informes de la red FABI señalan que, aunque existían grupos interesados en promover una revolución, «el movimiento de enero no fue planificado y en cierto modo fue precipitado por los acontecimientos espontáneos de la fábrica Vasena» (Díaz, 2019, p. 62). Las fuentes de inteligencia militar norteamericana, sin descartar una eventual instigación bolchevique a lo que era considerado no ya un levantamiento sino un estallido, estimaban las víctimas en 800 muertos y 2000 heridos, incluyendo muchas personas que no participaban de las protestas. ${ }^{15}$ Pocas semanas más tarde, el boletín semanal de inteligencia que recibía el presidente Wilson y las más altas autoridades norteamericanas, mostraba lo poco fundado del rumor que atribuía a los rusos la responsabilidad en la agitación de enero, apelando para ello a las cifras acerca de la nacionalidad de los más importantes dirigentes «maximalistas» de la Argentina, entre los cuales los originarios de Rusia eran solo ocho en un total de ciento dieciséis. ${ }^{16}$

Estas diferencias interpretativas no resultan en absoluto menores. Mientras la mirada del embajador Stimson, fuertemente influida por el temor a la revolución, coincidía en el diagnóstico del gobierno radical -al que sin embargo criticaba amargamente- y de la elite conservadora al denunciar un complot maximalista, la inteligencia norteamericana, articulada en la red FABI con sus aliados, conocía las dimensiones reales de las fuerzas revolucionarias y por ello consideró a la Semana Trágica como un episodio de protesta, en buena medida espontáneo y no asimilable a un levantamiento revolucionario.

\section{A modo de cierre}

La Semana Trágica tuvo consecuencias inmediatas en el plano represivo internacional. Gracias a las fuentes norteamericanas, sabemos que en Brasil «se tomaron severas medidas para evitar la llegada de anarquistas y otros indeseables.» ${ }^{17}$ No resulta casual que se haya realizado en Buenos Aires la Segunda Conferencia Internacional Sudamericana de

\footnotetext{
15 Biblioteca del Congreso de los Estados Unidos. Fondo Presidente Woodrow Wilson. Rollo de microfilm 471. Partes de inteligencia. Telegrama 1357 del 22 de enero de 1919. ${ }^{16}$ Biblioteca del Congreso de los Estados Unidos. Fondo Presidente Woodrow Wilson. Rollo de microfilm 469, Weekly Inteligence Sumary 91, 22 de febrero de 1919.

17 Biblioteca del Congreso de los Estados Unidos. Fondo Presidente Woodrow Wilson. Rollo de microfilm. Telegramas de inteligencia. Rollo 471.
} 
Policía, con el objetivo de buscar una fórmula eficiente de colaboración entre las fuerzas de seguridad de los países allí reunidos para, entre otros objetivos, controlar la circulación de personas con «cualidades antisociales» (Setemy, 2018, pp. 36-38).

Se tratan estas de consecuencias trasnacionales de un proceso que resultó a la vez local y trasnacional. En definitiva, estamos frente a una historia en la que la internacionalización de las luchas obreras se correspondió con la internacionalización del espionaje y la vigilancia policial, pero también con la circulación internacional de rumores, prejuicios y temores. En este sentido, en este trabajo hemos mostrado por un lado que el temor de las elites argentinas formaba parte de una corriente mucho más amplia que lo abarcaba; y, por el otro, que el temor a la revolución resultó un factor determinante para la comprensión de la política local por parte de la diplomacia norteamericana, cuya especificidad pudimos apreciar al compararla con la perspectiva de otros actores estadounidenses que -aunque fuertemente opuestos al maximalismoconstruyeron su información al margen de aquel terror.

\section{Referencias bibliográficas}

Avni, H. (1982). ¿Antisemitismo estatal en la Argentina ? (A propósito de los sucesos de la Semana Trágica - Enero de 1919). Coloquio, IV (8), 49- 67.

Babini, N. (1967). Pesadilla de una siesta de verano. La Semana Trágica. Todo es Historia, I (5), 10-23.

Bilsky, E. (1984). La Semana Trágica. Buenos Aires, Argentina: CEAL.

Díaz, H. (coord.). (2019). Espionaje y revolución en el Rio de la Plata. Los archivos secretos de una red diplomática de persecución al maximalismo (1918 - 1919). Buenos Aires, Argentina: CETHI-Imago Mundi.

Falcón, R. y Monserrat, A. (1998). Una vez más la Semana Trágica : estado de la cuestión y propuestas de discusión. Cuadernos del Ciesal. Revista de estudios multidisciplinarios sobre la cuestión social, 3 (4), 35-50.

Godio, J. (1986[1972]). La Semana Trágica de enero de 1919. Buenos Aires, Argentina: Hyspamérica. 
Lefebvre, G. (1982 [1932]). The Great Fear of 1789. Rural panic in Revolutionary France. Princeton, Estados Unidos: Princeton University Press.

Lvovich, D. (2003) Nacionalismo y Antisemitismo en la Argentina. Buenos Aires, Argentina: Ediciones B.

Lvovich, D. (2016). La Semana Trágica en clave trasnacional. Influencias, repercusiones y circulaciones entre Argentina, Brasil, Chile y Uruguay, 1918-1919. En Bertonha, J. y Bohoslavsky, E. (Comps). Circule por la derecha. Diálogos, redes y trayectorias entre las derechas sudamericanas, 1917-1973. Buenos Aires, Argentina: UNGS.

Mirelman, V. (1975). The Semana Trágica of 1919 and the jews in Argentina. Jewish Social Studies, (37), 61-72.

Murray, R. (1955). Red Scare: A Study in National Hysteria, 1919-1920. Minneapolis, Estados Unidos: University of Minnesota Press.

Oved, I. (1978). El anarquismo y el movimiento obrero en Argentina. México, México: Siglo XXI.

Rock, D. (1972). Lucha Civil en la Argentina. La Semana Trágica de enero de 1919. Desarrollo Económico, (42-44), 165-215.

Schmidt, R. (2000). Red Scare: FBI and the Origins of Anticommunism in the United States, 1919-1943. Copenhage, Dinamarca: Museum Tusculanum Press-University of Copenhage.

Seibel, B. (1999). Crónicas de la Semana Trágica. Buenos Aires, Argentina: Corregidor.

Setemy, A. (2018). Sentinelas das fronteiras. A diplomacia brasileira e a conexaorepressiva internacional para o combate ao comunismo. Curitiba, Brasil: Prismas.

Solominsky, N. (1971). La Semana Trágica en la Argentina. Buenos Aires, Argentina: Biblioteca Popular Judía del Congreso Judío Mundial.

Stimson, F. (1931). My United States. Nueva York/Londres, Estados Unidos/Inglaterra: Charles Scribner's sons.

Tur Donati, C. (1993). Crisis Social, xenofobia y nacionalismo en Argentina, 1919. Cuadernos Americanos, 6 (42), 38-77. 